\title{
Automation and Molecular Diagnostics: A New Era in Clinical Microbiology
}

\begin{abstract}
The environment of the clinical microbiology laboratory is rapidly changing. Testing methodologies based upon organism growth on an array of liquid and solid media are being replaced by newer methods that enhance the rate of organism identification as well as increase the sensitivity and specificity by which identification occurs. A majority of these new techniques are based upon nucleic hybridization and polymerase-chain reaction technology and range from identification of single-organisms or organism families to multiplexed syndromic panels, which can concurrently examine for the presence of numerous suspect organisms based upon the symptoms exhibited by the patient. In addition, the clinical microbiology laboratory now has access to a level of automation thus far only seen in the chemistry and hematology sections of the clinical laboratory. These transitions have been repeatedly shown to enhance the level of patient care when properly implemented into the laboratory workflow. Conversely, with the rapid encroachment of these new technologies comes potential downfalls, including cost and challenges with training laboratory staff. Collectively, the clinical microbiology laboratory is coming into a new era of technology and patient care that will bring about dramatic changes to conventional testing and organism identification.
\end{abstract}




\section{LEARNING OBJECTIVES:}

1. Describe the three categories of nucleic acid-based testing.

2. Describe the currently available and developing technologies for the clinical microbiology lab, naming specific examples and the pros and cons of their implementation and utilization.

3. Describe the impact these newly implemented and future technologies may have on clinical microbiology workflow, personnel training, and the education of future laboratory professionals. 


\begin{abstract}
ABBREVIATIONS:
BCID - Blood culture identification, BD - Becton Dickinson, CDC - Centers for Disease Control and Prevention, CLIA - Clinical Laboratory Improvement Amendments, CMS - Centers for Medicare and Medicaid Services, CNS - central nervous system, FDA - Food and Drug Administration, FDA-ARGOS - Food and Drug Administration-dAtabase for Regulatory-Grade micrObial Sequences, FISH - fluorescent in-situ hybridization, GI - gastrointestinal, HAD helicase-dependent amplification, HIV - human immunodeficiency virus, LAMP - loop-mediated isothermal amplification, MALDI-TOF - matrix assisted laser desorption ionization-time of flight, MRSA - methicillin resistant Staphylococcus aureus, NGS - next-generation sequencing, PCR - polymerase chain reaction, POC - point of care, RNA - ribonucleic acid, STI - sexually transmitted infection, TLA - total laboratory automation, TMA - transcription-mediated amplification, VRE - vancomycin resistant Enterococcus, WASP - Walk-away Specimen Processor
\end{abstract}




\section{INDEX TERMS:}

Molecular diagnostics, syndromic panels, next-generation sequencing, total laboratory automation 


\section{INTRODUCTION}

The clinical microbiology lab has been witness to rapid advancements in technology and automation in recent years. Culture-based testing methodologies remain the "gold standard" for most laboratories but there have been several technological advances incorporated that decrease result turnaround time and subsequently enhance patient care. The previous article for this Focus Series discussed at length the biochemical and traditional testing that is still a large component of organism identification as well as the increasing incorporation of matrix assisted laser desorption ionization-time of flight (MALDI-TOF) technology. For this article, these methods will be referred to as conventional testing. The term conventional encompasses essentially all culturebased methods that require a pure isolated colony for testing to occur. Although not a conventional method as of yet, MALDI-TOF technologies still rely upon pure isolates and will only be briefly discussed.

In this review, we will focus on recent advances in molecular diagnostics that have entered the microbiology lab, including single-target polymerase chain reaction (PCR)-based and nucleic acid hybridization-based assays to the newest syndromic panels that are rapidly receiving Food and Drug Administration (FDA) approval. We will also discuss how this technology is being incorporated into point of care (POC) testing, classified as CLIA-waived, and the associated advantages and pitfalls. Lastly, we will look into the future of the clinical microbiology lab with the potential incorporation of next-generation sequencing (NGS) and metagenomics for organism identification as well as advancements in microbiology total laboratory automation (TLA). With all of the recent advances, there are many corporations 
developing new testing platforms that are rapidly receiving FDA-approval, or will so in the near future. In light of this, literature searches demonstrate many comprehensive reviews discussing these new technologies, giving very detailed comparisons between the platforms and making compelling arguments for the pros and cons of each. The scope of this review is limited and geared toward a general audience and therefore cannot acknowledge every advancement or piece of literature. Any mention of specific technologies, scientific studies, or reviews is in no way an endorsement of one over another.

\section{SINGLE TARGET MOLECULAR DIAGNOSTICS}

The development of PCR by Cary Mullis in $1985^{1}$ is arguably one of the biggest contributions to science and modern medicine. The principles behind this technique have allowed science to decipher the genomes of many species and serves as the basis for the field of molecular diagnostics. ${ }^{2}$ Some of the first molecular tests to become FDA-approved and introduced into the clinical microbiology laboratory were single-target tests that utilize PCRbased amplification techniques. These assays were followed by technique modifications such as transcription-mediated amplification (TMA), loop-mediated isothermal amplification (LAMP), and helicase-dependent amplification (HDA), to name a few. ${ }^{3}$ These single-target molecular tests also utilize signal amplification and hybridization techniques. ${ }^{4}$ One of the first areas of the clinical lab to greatly benefit from these advancements was the virology section. Molecular testing for viral pathogens in patient samples made way for the elimination of tedious, contamination-prone viral cultures using mammalian cell systems. These culture methods could take weeks for a positive result, if any viable virus could be propagated at all. ${ }^{5}$ Culture-based assays would also require the clinical microbiology laboratory to have a designated biosafety 
cabinet, incubators, and reagents specific for mammalian cell culture. ${ }^{6}$ Introduction of molecular diagnostics eliminated this need and allowed for determination of viral presence directly from patient specimens with results determined in hours instead of days/weeks. ${ }^{5}$ Arguably one of the largest impacts molecular testing has had on patient and public health is detecting human immunodeficiency virus (HIV). The rapidness and sensitivity of molecular tests allow for early detection of the virus so that proper treatment regimens may be started sooner and, from the public health standpoint, provide the patient with the knowledge of their HIV status which helps in the prevention of the spread of the disease. ${ }^{5}$ Another example is the introduction of PCRbased testing for influenza A and B. ${ }^{7}$ These influenza tests give very rapid results to the ordering physician so that patients may be started on appropriate antiviral therapies while also providing valuable epidemiological information about the prevalence of the virus during yearly flu seasons. As can be seen on the FDA website ${ }^{8}$, there are multiple testing platforms and kits that allow for the direct detection of viral presence. Of the PCR-based technologies listed, all have their specific pros and cons mainly surrounding variability in sensitivity and specificity ${ }^{2}$, but collectively all greatly improve upon patient care. Selection of the ideal platform is a lab-specific determination that must be based upon technical expertise as well as space and budget constraints.

Another area of microbiology testing that has benefitted from molecular advancements is the identification of sexually transmitted infections (STIs). ${ }^{2}$ STIs are noted public health issues ${ }^{9}$ and some of the causative organisms are notoriously difficult or impossible to culture in standard laboratory settings (i.e. Chlamydia trachomatis and Trichomonas vaginalis). Others, such as Neisseria gonorrhoeae can cause asymptomatic infection, especially in women, leading to missed diagnoses. Using molecular-based testing for suspected STIs or STI screening, especially 
for individuals at higher risk or who engage in high risk behaviors, greatly benefits public health due to more rapid testing and result reporting. ${ }^{2,10}$

These single-target assays have also been of great use to patient/hospital management in terms of identification of drug resistant organisms (methicillin-resistant Staphylococcus aureus (MRSA), vancomycin-resistant Enterococci (VRE), etc.) and causes of nosocomial infections, such as Clostridioides difficile, which typically leads to patients being placed in isolation. ${ }^{11}$ Recognition of these pathogens in a timely manner helps prevent spread of these organisms throughout patient-care areas. Conversely, it also helps prevent unnecessary isolation procedures, leading to reduced hospital costs. ${ }^{12}$

A summary of the number of available FDA-approved molecular tests for single organisms or organism groups (i.e. same genus or subtypes) can be found in Table 1.

\section{POINT OF CARE MOLECULAR DIAGNOSTICS}

As we see with other methods employed in clinical microbiology laboratories (and other laboratory sections), once an assay or technique has been thoroughly vetted and validated the move to simplify these assays and reduce laboratory hands-on time and overall costs is possible. A majority of point of care (POC) testing in the past has been of the immunochromatography variety ${ }^{13}$ but the introduction of POC molecular testing is starting to come to the forefront due to "PCR-in-a-box" technology which utilizes a closed system, thus greatly reducing the potential for contamination and therefore aberrant or incorrect results. ${ }^{4}$ This type of testing has already been employed in the form of influenza A and B and Group A Streptococcus testing. ${ }^{13}$ It is easy to see the utility of these types of tests in a POC setting, such as walk-in clinics. Patients may receive their results while still in the examination room (in most cases), allowing rapid 
progression from diagnosis to prescribed treatment. A major benefit of this rapid testing is the minimization of an afflicted individual's contact with other people. Another benefit is that POC tests are designed to be essentially foolproof by being very simplistic in design and procedure and unlikely to give false-results, thus allowing non-laboratory personnel to run these tests with success. ${ }^{14}$ This, however, does give cause for concern and requires a watchful eye as more CLIA-waived tests come to the forefront due to the fact that they oftentimes lack accredited laboratory professionals performing regular quality control and assurance.

\section{SYNDROMIC PANELS}

Syndromic panels are exciting new laboratory tools that are actively being used in many clinical microbiology laboratories. These multiplexed panels are a very hot topic in clinical microbiology and extensive reviews and studies have been published describing their design and clinical performance. ${ }^{15-20}$ We will briefly cover the highlights and capabilities of these systems.

Multiplexed panels are referred to as "syndromic" as they are designed to test for a battery of organisms (viral, bacterial, fungal, and parasitic) that are most commonly associated with a specific set of symptoms exhibited by the patient. A major advantage of these systems, that is attributed to their rapid introduction into the clinical lab, is testing is performed directly from patient samples without the need for culturing to isolate organisms. To date, panels for sepsis, respiratory infections, gastrointestinal infections, and central nervous system (meningitis/encephalitis) infections are approved for clinical use. ${ }^{15}$ Biofire Diagnostics' FilmArray (or FilmArray Torch) and Luminex Corporation's Verigene systems have panels for the syndromes listed above. Genmark Diagnostic's eSensor and ePlex systems offer a respiratory panel. 


\section{Sepsis}

The search for a sensitive, rapid means of identification of organisms from patients suspected of being septic is a major need in healthcare. The Centers for Disease Control and Prevention (CDC) reports that 1 of 3 patients who die in a hospital have sepsis. ${ }^{21}$ When a patient is admitted for sepsis, empiric antibiotic therapy is often administered until a specific organism is identified and susceptibilities are given. Most blood culture detection systems require some incubation time and identification relies upon conventional testing. To this end, blood culture panels have been designed to identify organisms from positive blood culture bottles. While the laboratory must still wait for blood cultures to become positive, these panels will expedite the identification process compared to conventional methods by allowing identification within 1-2.5 hours, depending on the organism and system used. ${ }^{15}$

The BioFire FilmArray BCID system can identify 27 bacterial and yeast pathogens with one assay that takes approximately 1 hour to complete. The Verigene system has two separate panels, one for gram-positive bacteria and one for gram-negative, that can detect 15 and 14 organisms within 2-2.5 hours, respectively. ${ }^{15,17}$ Both systems, within the panel runtime, are able to provide information on the presence of antibiotic resistance genes within isolates. ${ }^{15,17}$ While this is not indicative of active antibiotic resistance, it may offer some guidance in the usage of antibiotics. True antibiotic sensitivities must still be collected through conventional testing. Collectively, these systems allow tailored antibiotic administration earlier (instead of empiric) and subsequently deliver better clinical outcomes. ${ }^{22-25}$

There are non-FDA approved panels in development that are able to test blood samples directly, without prior incubation. One such system is the T2Candida panel from T2 Biosystems. This assay has been shown to have similar sensitivity for the identification of five separate 
Candida species in conventional blood cultures. ${ }^{26,27}$ This is a promising development for patients suspected of being septic, especially if this system or others like it can be modified to accommodate testing for a variety of other microorganisms.

Although not a syndromic panel-type platform as described above, a hybridization-based technology named the Accelerate Pheno System has been recently developed for blood cultures by Accelerate Diagnostics. This system, using fluorescent in situ hybridization (FISH) technology and automated single-cell microscopy can detect gram-positive and gram-negative organisms directly from blood cultures and determine their antibiotic sensitivities by utilizing a continuous colony growth monitoring system. ${ }^{15}$

\section{Respiratory Infections}

There is more variety among available respiratory infection panels compared to the other panels discussed. All FDA-approved panels are multiplexed, utilize unique molecular-based detection systems, and offer quick turnaround times of one to eight hours. ${ }^{15,18}$ They also all utilize nasopharyngeal swabs as the specimen of choice. Respiratory pathogen panels are offered on the BioFire FilmArray, Verigene/Luminex systems, and GenDiagnostic's eSensor and ePlex systems. Each array offers their own variety of viral and bacterial respiratory pathogens and tests for a select sampling of bacteria that are notoriously challenging to culture (ex. Chlamydia pneumoniae, Mycoplasma pneumoniae, and Bordetella spp.). Compared to conventional assays, multiplexed testing for respiratory pathogens decreases the time to identification and thus, lowered hospital admission rates, fewer chest X-rays, shorter hospital stays, and decreased

duration of antimicrobial treatment (de-escalation of antibiotics). ${ }^{28,29}$ The latter outcome is very important for antimicrobial stewardship as patients who arrive with respiratory illness symptoms that do not point to a specific diagnosis may be administered antibiotics as a precaution. Rapid 
identification of a viral infection leads to elimination of unnecessary antibiotic therapy, if initially started, thereby preventing the potential development of or selection for drug-resistant organisms.

Within the past year, BioFire released a pneumonia panel that specifically targets organisms typically found in lower respiratory tract infections. In contrast to the respiratory infection panels previously discussed, this pneumonia panel utilizes sputum or bronchoalveolar lavage specimens. Collectively, this panel can simultaneously report results for 33 separate targets including 26 pathogens (18 bacterial and 8 viral) and 8 antimicrobial resistance genes within one hour of startup. ${ }^{30}$ Given the recent FDA-approval for this panel, there have been no published clinical studies comparing the sensitivity and specificity of this panel to conventional culture, but there is a study currently in early stages. ${ }^{31}$ This panel will likely serve to fill a gap in the clinical microbiology lab as a recent study demonstrated the efficacy of using the BioFire FilmArray BCID panel to test for organisms causing ventilator-associated pneumonia ${ }^{32}$, which is a non-FDA approved use of this panel.

\section{Gastrointestinal Infections}

Another available panel that has achieved FDA-approval is designed to potentially identify the causative agent(s) of infectious diarrhea. This is a rapid way to detect the most likely suspected organisms as identification through conventional testing can require days. The Verigene system can detect a total of 9 targets ( 7 bacteria and 2 viral) and is scalable from one to 32 samples with a run time of less than two hours. The Magpix/Luminex system can detect 20 separate pathogens (14 bacterial, 3 viral, and 3 parasitic) and is capable of running 24 samples at a time. This system is not scalable and takes around 5 hours to complete the assay. Lastly, the BioFire FilmArray system/FilmArray Torch is capable of targeting 22 pathogens (13 bacterial, 5 
viral, and 3 parasitic) and is scalable from 1-12 assays which take about one hour to reach completion. $^{15}$

Advantages seen with these panels are that they collectively demonstrate a high sensitivity and specificity to the selected targets, with only a few exceptions, and typically catch organisms that are missed with conventional testing. ${ }^{33-36}$ These panels can also quickly deliver information about whether or not a patient should be put in isolation procedures. This is critical in preventing the spread of nosocomial infections as well as saving costs by removing patients from isolation procedures days before conventional testing would allow.

The big question that remains (as with all panels) is how these GI panels will perform in day-to-day clinical microbiology labs. One caveat with these types of panels is that once identification is made there is not an isolated colony for additional susceptibility testing and therefore no organism to send to state public health laboratories, as is necessary with organisms such as Salmonella spp., Shigella spp., or Escherichia coli O157:H7. ${ }^{37,38}$ Another issue reported is that many assays will return multiple-target (two or more) positive results in around $16 \%$ of samples tested ${ }^{33}$. This indicates that precaution must be taken with interpreting results and that the overall patient condition and symptoms must be closely examined in conjunction with panel results. For example, in a scenario where two or more pathogens may be encountered, a positive result for $C$. difficile may have a very different meaning for a patient with community acquired diarrheal disease versus a patient who has been hospitalized. In this scenario, $C$. difficile may very well be part of the normal GI flora. ${ }^{15}$ Again, the entire clinical scenario must be taken into account with these types of situations and resist treating indiscriminately for every organism that gives a positive result.

\section{Central Nervous System Infections}


Patients suffering from meningitis and encephalitis are in danger of facing devastating outcomes associated with high morbidity and mortality rates. ${ }^{39}$ Upon presentation of symptoms, such as altered mental/neural states, headaches, light sensitivity, etc., there is an urgent need for rapid diagnosis of the infectious agent. Prior to 2015, there were several stand-alone PCR-based tests for potential viral agents that can cause these diseases but none for bacterial or fungal organisms. ${ }^{15}$ Adding to the potential delay in treatment, conventional methods of identification for bacteria and fungi, again, potentially take days. Confounding this method even further is that conventional culturing methods may come back negative (no growth) if a patient has been placed on empiric antibiotic therapy immediately upon arrival with the aforementioned symptoms.

Biofire's FilmArray Meningitis/Encephalitis panel was the first to offer multiplexed viral, bacterial, and fungal assays for the most commonly encountered agents of central nervous system (CNS) infections. This panel tests for 14 different pathogens (7 viral, 6 bacterial, 1 fungal) and results are returned in roughly an hour with only two minutes of hands-on time by the laboratory staff. ${ }^{15}$ The advantages of this panel are similar to the others discussed in that it shows a higher sensitivity and specificity for the target organisms compared to conventional testing. ${ }^{40-44}$ It can also detect bacterial and fungal organisms after antibiotic/antifungal therapies have begun. ${ }^{15}$ In terms of antimicrobial stewardship, similar to other panels, confirmation of a viral pathogen causing the CNS infection allows discontinuing antimicrobial therapy sooner. ${ }^{15}$

The drawbacks to the panel reflect its utility in everyday clinical use. Situational awareness with the ordering physician in interpreting data is crucial. Many of the organisms that can be detected are becoming more rare thanks to immunizations (Haemophilus influenzae, Neisseria meningitidis). It may also be difficult for this assay to supplant some current rapid 
testing already performed in the lab, such an example is testing of cerebrospinal fluid for Cryptococcus neoformans antigen. ${ }^{45-47}$

\section{Syndromic Panel Summary}

Overall, multiplexed nucleic acid detection panels offer several advantages for diagnosing infections in the clinical microbiology lab. Clinical data shows that the panels are superior to conventional methods in both sensitivity and specificity. ${ }^{22-25,28,29,33,36,40-44}$ The risk for contamination from the laboratory setting is also low since these assays employ closed systems; however, aberrant or discrepant results must be scrutinized along with regular control testing to rule out potential system contamination as there are studies indicating this can be a concern with

certain panels. ${ }^{40}$ Currently, there are studies using existing panels to test other sterile fluid samples (ex. synovial or pleural fluids) for the presence of infectious organisms and the reports are showing some success with this approach (although not an FDA-approved panel usage). ${ }^{48,49}$ There are also FDA-approved panels used for military and bioterrorism purposes that test for the most common organisms that could be encountered in biological warfare. ${ }^{8}$ These panels also have the major benefit of identifying infectious agents in 1-5 hours, depending on which panel/platform is used. This rapid turnaround time has major benefits to patient care in terms of the beginning (or stopping) of treatments and isolation practices and, in some instances, promoting shorter hospital stays. ${ }^{15,16,18}$

Conversely, a major issue with these panels is implementation into clinical practice. The panels are associated with a high cost and the potential for non-reimbursement from the Centers for Medicare and Medicaid Services (CMS). ${ }^{45}$ It can be argued that these costs per test be offset by the money saved through more appropriate patient treatment leading to shorter hospital stays. ${ }^{15}$ This is, of course, if the panels are used appropriately and lead to the successful 
identification of the infectious agent. Panels are also not typically customizable so if the organism present is not on the list, then it will not be detected. Despite the rapidness of these assays and the fact they detect organisms directly from patient samples, they leave no isolated colonies for subsequent susceptibility studies for which conventional methods are still the gold standard. Although some are able to detect antibiotic resistance genes within the patient sample, two major caveats exist. The first being that the presence of a resistance gene does not necessarily dictate that an organism will display functional resistance to a particular antimicrobial. $^{50,51}$ Secondly, in the instance of coinfections, the presence of a resistance gene cannot be attributed to a specific organism.

For these panels to have optimal success, communication is key between the ordering physician and the laboratory. These panels may lose their diagnostic potential very rapidly if they are viewed as a screening test where one can probe to see what might be present with no supportive reasoning. For optimal implementation, it will require assessment of local epidemiological factors, such as particular disease incidence rates, to determine which panels would be appropriate for use. In other words, this assay should not ideally be used to test for rarely encountered organisms as a first step in diagnosis as it is of high importance that proper workflow assessments be made so that these tests are appropriately utilized only when there would be a direct benefit to patient care and cost effectiveness.

\section{NEXT GENERATION SEQUENCING}

One of the newest technologies developed is next generation sequencing (NGS) which is accompanied by subsequent metagenomics analyses. NGS is promising in that it can identify every organism in a patient specimen without requiring specific probes or primer sets. ${ }^{52}$ NGS 
technologies sequence small segments of the entire microbial genome and then compare the sequences with established databases for identification. Pyrosequencing, one of the first NGS technologies developed, allows entire sequencing of $16 \mathrm{~S}$ ribosomal RNA as well as whole genomes of microbes. ${ }^{52}$ Since then, other methods have been introduced that enhance NGS diagnostics. $^{52}$

The massive amount of information collected from whole-genome sequencing or the tailored identification of every organism in a biofilm or the gut biome is staggering, but exciting, in terms of the diagnostic information that can be obtained and used for patient care. This technology can deliver significantly more information on an isolate or disease state than any current identification method in the clinical microbiology lab, including multiplexed nucleic acid detection and MALDI-TOF. NGS techniques can identify and differentiate organism serotypes, antibiotic resistance genes, as well as provide additional genomic detail about an organism that may be useful. ${ }^{52-57}$ The FDA is in the process of creating FDA-ARGOS (FDA-database for regulatory-grade microbial sequences), which is a regulatory grade database for microbial sequences that would be the reference standard to which NGS-generated diagnostic sequences would be compared. ${ }^{58}$

Despite the powerful analytical capabilities of NGS, there are many caveats that act as a hindrance to its incorporation into clinical microbiology labs. Firstly, the lack of FDA-approval is a significant hurdle; however, as just discussed, this should be alleviated in the near future once the FDA-ARGOS database is put into clinical use. Secondly, NGS in its current state is very expensive and time-consuming compared to already available technologies, such as multiplexed PCR testing. ${ }^{59}$ Additionally, since NGS tests patient samples directly, therein lies the challenge of determining which organisms are colonizers/normal flora or are present in a 
pathological state. This would be even more challenging in the setting of an immunocompromised patient, where the expected colonizer can very well be the source of disease. There is also the obstacle of how laboratories can implement and validate an instrument that can pick up any and all known pathogens, whereas current molecular testing utilizes preestablished groups of target organisms. ${ }^{60}$ As technologies improve and become more streamlined, associated costs become more justified, and regulatory standards are set, the ability to incorporate NGS in clinical labs will start to become more of a reality. ${ }^{52,58,59-63}$

\section{TOTAL LABORATORY AUTOMATION}

Total laboratory automation (TLA) and robotics have been a part of the clinical lab (especially chemistry and hematology) for decades; however, throughout this time, the microbiology lab has not progressed very much in this area. There is stand-alone automation in the microbiology lab, such as blood culture and identification systems (discussed in the previous Focus Series article) but the idea of unified TOTAL automation, from specimen processing to release of patient results, is a fairly new concept. A large reason for this is the variety of specimen types processed in the clinical microbiology lab. This variety has prevented a "one size fits all" approach to sample processing and distribution for culture, which would be a cornerstone for automation.

Liquid transport media has been suggested as a solution to specimen standardization ${ }^{64}$ for total automation that also works well with the newest identification techniques in the microbiology lab, such as MALDI-TOF and multiplexed PCR. ${ }^{65}$ The push for TLA is also driven by the oftentimes understaffed laboratory that is handling increased volumes of patient specimens sent to the laboratory for testing. ${ }^{65}$ Collectively, this makes automation very 
appealing as it can free up valuable time from what some may consider mundane or repetitive tasks such as media inoculation.

Currently, there are two systems for TLA in clinical microbiology, the BD Kiestra and the COPAN Diagnostics WASPLab system. Both systems can store, label, and inoculate multiple types of media with samples and include a track system that will transport the inoculated plates to smart incubators. ${ }^{66}$ Multiple studies have demonstrated that automating specimen inoculation alone can dramatically increase the quality and numbers of individually isolated colonies. ${ }^{67-71}$ Additionally, automation may enhance the number of fastidious organisms isolated from urine samples. $^{72}$

The aptly-named smart incubators continuously monitor agar plate media for organism growth by using high resolution imaging at regularly-timed intervals. These images can then be viewed by laboratorians at a workbench display where decisions can be made regarding further workup. The $\mathrm{BD}$ Kiestra is also able to deliver the plates, upon request, to the workbenches should the laboratory scientist want to inspect them manually. ${ }^{66}$

The huge advantage of this type of automated analysis is that colony images can be shared with other labs, colleagues, and specialists around the globe. High-resolution imaging also leads to earlier single colony detection which may be sufficient for identification via MALDI-TOF systems. ${ }^{66}$ A thorough review by Croxatto et al. ${ }^{66}$ describes in detail the similarities and differences in technical specifications between the BD Kiestra and COPAN WASPLab systems.

There are a number of obstacles in the way concerning the ubiquitous adoption of total automation in diagnostic laboratories, primarily the cost and size of the units. These automated laboratories are quite expensive and require a large amount of space that may not be feasible to 
obtain with many current hospital infrastructures. ${ }^{65}$ Another issue is that they are not inclusive of every culture and specimen type. Also, in the case of a system or software crash or malfunction, backup processing and testing must still be completed. Overall, it is exciting to imagine this technology being incorporated into everyday clinical microbiology labs, but the reality is that it may not be ready for widespread use yet. It will take much deliberation to determine the best course of action for any particular laboratory or laboratory network to assess the feasibility of incorporating TLA.

\section{DISCUSSION}

Clinical microbiology laboratories are going through very exciting changes. New technologies and vast improvements to existing technology are rapidly improving patient care and decreasing patient costs through reduction of the time it takes to identify infectious agents causing disease. Currently, diagnostic microbiology seems to be at a crossroads between conventional testing and implementation of advanced testing platforms and full-scale automation. Admittedly, there are concerns surrounding this explosion of advanced technology. Laboratory directors and managers must scrutinize and decide what works best for their laboratory environment because a small rural hospital, if they still maintain a fully functioning microbiology lab at all, is likely not going to see the same volume as a larger hospital in an urban center. Therefore, the rural locale would likely not benefit as greatly by moving to a fully automated laboratory or incorporating the bulk of current and future advanced technology.

There is also concern that the new testing panels, with all of their advantages, will lure physicians into ordering tests that are unnecessary and therefore potentially increase patient and laboratory costs. ${ }^{15}$ On top of this, there is the issue of so much information being produced from 
these tests that interpretation of the actual meaning of the results could be made more difficult or altogether lost in translation. This could potentially lead to poorer patient outcomes as well as reversion to conventional methods in order to provide answers the panel could not provide. These are issues to take into consideration when incorporating new technology into existing laboratory workflows.

From an educational standpoint, these advances pose a conundrum in both the workforce and in the classroom. It has been estimated that the average age of a clinical microbiologist is over 50 years old. ${ }^{65}$ This indicates that much of the current workforce has been taught, trained, and certified in conventional testing methodologies. This puts the onus on existing microbiologists to become competent and demonstrate expert judgement with technology introduced into the laboratory rather than assuming the "set it and forget it" style of newer technology platforms is error-free, and therefore taking all results at face value. In the classroom setting, the challenge lies with balancing the curriculum to match what is happening in the clinical labs. This leads to nearly double the amount of educational material as clinical laboratory educators are still currently held to the standard of teaching conventional methods as well as the principles behind the new technology that our graduates may encounter early on in their careers. As such, educators now faced with the decision of whether to shift their curriculum further toward PCR-based and MALDI-TOF identification over conventional identification methods or double course content. Regardless of the path taken, the wheels of technology are spinning fast and watching the effects this has on clinical microbiology laboratories will be filled with a healthy mix of skepticism and wonder.

The technological landscape of the clinical microbiology lab is rapidly evolving and does not appear to be slowing in this aspect. In just a few decades, clinical microbiologists have 
witnessed the advances from solely culture-based, manual biochemical testing to automated biochemical testing to the incorporation of MALDI-TOF organism identification. In addition to these impactful advances, PCR-based testing has already deeply rooted itself into clinical microbiology testing. A key advancement associated with molecular testing is that it can be performed directly on patient samples without the need for isolation of pure bacterial colonies, enhancing time to result and subsequently, patient care. Even now, current FDA-approved molecular assays continue to be improved upon in terms of sensitivity and number of targets in conjunction with the development of new assays. The excitement brought on by these changes is further compounded by the diagnostic potential for the next era of technological progress, such as NGS and total laboratory automation. It will be thrilling to see what the future holds for the clinical microbiology laboratory. 


\section{REFERENCES}

1. Mullis K, Faloona F, Scharf S, Saiki R, Horn G, Erlich H. Specific enzymatic amplification of DNA in vitro: the polymerase chain reaction. Cold Spring Harbor Symp Quant Biol $1986 ; 51: 263-73$

2. Fairfax MR, Bluth MH, Salimnia H. Diagnostic molecular microbiology: A 2018 snapshot. Clin Lab Med. 2018;38(2),253-276

3. Buchan BW, Ledeboer NA. Emerging technologies for the clinical microbiology laboratory. Clin Microbiol Rev. 2014;27(4):783-822

4. Das S, Shibib DR, Vernon MO. The new frontier of diagnostics: Molecular assays and their role in infection prevention and control. Am J Infect Control. 2017;45(2),158-169.

5. Josko D. Molecular virology in the clinical laboratory. Clin Lab Sci. 2010;23(4),231-6.

6. Hematian A, Sadeghifard N, Mohebi R, Taherikalani M, Nasrolahi A, Amraei M, et al. Traditional and modern cell culture in virus diagnosis. Osong Public Health Res Perspect. 2016;7(2),77-82. 
7. Wabe N, Li L, Lindeman R, Yimsung R, Dahm MR, Clezy K, et al. The impact of rapid molecular diagnostic testing for respiratory viruses on outcomes for emergency department patients. Med J Aust. 2019;210(7),316-320.

8. FDA Nucleic Acid Based Tests-Microbial Tests [Internet]. U.S. Food and Drug Administration; c2019 [cited 2019 July 12]. Available from https://www.fda.gov/medicaldevices/vitro-diagnostics/nucleic-acid-based-tests

9. Low N, Broutet N. Sexually transmitted infections-research priorities for new challenges. Plos Med. 2017;14(12):e1002481.

10. Cristillo AD, Bristow CC, Peeling R, Van Der Pol B, de Cortina SH, Dimov IK, et al. Pointof-care sexually transmitted infection diagnostics: Proceedings of the star sexually transmitted infection-clinical trial group programmatic meeting. Sex Transm Dis. 2017;44(4),211-218.

11. Josko D. Molecular bacteriology in the clinical laboratory. Clin Lab Sci. 2010;23(4):237-41.

12. Sewell B, Rees E, Thomas I, Ch'ng CL, Isaac M, Berry N. Cost and impact on patient length of stay of rapid molecular testing for clostridium difficile. Infect Dis Ther. 2014;3(2):281-293.

13. Kozel TR, Burnham-Marusich AR. Point-of-care testing for infectious diseases: Past, present, and future. J Clin Microbiol. 2017;55(8):2313-2320.

14. FDA CLIA Categorizations [Internet]. U.S. Food and Drug Administration; c2018 [cited 2019 July 12]. Available from https://www.fda.gov/medical-devices/ivd-regulatoryassistance/clia-categorizations

15. Ramanan P, Bryson AL, Binnicker MJ, Pritt BS, Patel R. Syndromic panel-based testing in clinical microbiology. Clin Microbiol Rev. 2017;31(1).

16. Patel R. New developments in clinical bacteriology laboratories. Mayo Clin Proc. 2016;91(10):1448-1459. 
17. Ward C, Stocker K, Begum J, Wade P, Ebrahimsa U, Goldenberg S. Performance evaluation of the Verigene ${ }^{\circledR}$ (Nanosphere) and FilmArray ${ }^{\circledR}\left(\right.$ BioFire $\left.{ }^{\circledR}\right)$ molecular assays for identification of causative organisms in bacterial bloodstream infections. Eur J Clin Microbiol Infect Dis. 2015;34(3):487-96.

18. Hanson KE, Couturier MR. Multiplexed molecular diagnostics for respiratory, gastrointestinal, and central nervous system infections. Clin Infect Dis. 2016;63(10):1361-1367.

19. Huang RS, Johnson CL, Pritchard L, Hepler R, Ton TT, Dunn JJ. Performance of the Verigene ${ }^{\circledR}$ enteric pathogens test, Biofire FilmArray ${ }^{\mathrm{TM}}$ gastrointestinal panel and Luminex $\mathrm{xTAG}{ }^{\circledR}$ gastrointestinal pathogen panel for detection of common enteric pathogens. Diagn Microbiol Infect Dis. 2016;86(4):336-339.

20. Popowitch EB, O'Neill SS, Miller MB. Comparison of the Biofire FilmArray RP, Genmark eSensor RVP, Luminex xTAG RVPv1, and Luminex xTAG RVP fast multiplex assays for detection of respiratory viruses. J Clin Microbiol. 2013;51(5):1528-33.

21. CDC Sepsis Data \& Reports [Internet]. Centers for Disease Control and Prevention; c2016 [cited 2019 July 12]. Available from https://www.cdc.gov/sepsis/datareports/index.html

22. Suzuki H, Hitomi S, Yaguchi Y, Tamai K, Ueda A, Kamata K, et al. Prospective intervention study with a microarray-based, multiplexed, automated molecular diagnosis instrument (Verigene system) for the rapid diagnosis of bloodstream infections, and its impact on the clinical outcomes. J Infect Chemother. 2015;21(12):849-56.

23. Beal SG, Thomas C, Dhiman N, Nguyen D, Qin H, Hawkins JM, et al. Antibiotic utilization improvement with the Nanosphere Verigene Gram-Positive Blood Culture assay. Proc (Bayl Univ Med Cent). 2015;28(2):139-43. 
24. Walker T, Dumadag S, Lee CJ, Lee SH, Bender JM, Cupo Abbott J, et al. Clinical impact of laboratory implementation of Verigene BC-GN microarray-based assay for detection of gramnegative bacteria in positive blood cultures. J Clin Microbiol. 2016;54(7):1789-1796.

25. Bork JT, Leekha S, Heil EL, Zhao L, Badamas R, Johnson JK. Rapid testing using the Verigene gram-negative blood culture nucleic acid test in combination with antimicrobial stewardship intervention against Gram-negative bacteremia. Antimicrob Agents Chemother. 2015;59(3):1588-95.

26. Pfaller MA, Wolk DM, Lowery TJ. T2MR and T2Candida: novel technology for the rapid diagnosis of candidemia and invasive candidiasis. Future Microbiol. 2016;11(1):103-117

27. Mylonakis E, Clancy CJ, Ostrosky-Zeichner L, Garey KW, Alangaden GJ, Vazquez JA, et al. T2 magnetic resonance assay for the rapid diagnosis of candidemia in whole blood: A clinical trial. Clin Infect Dis. 2015;60(6):892-9.

28. Subramony A, Zachariah P, Krones A, Whittier S, Saiman L. Impact of multiplex polymerase chain reaction testing for respiratory pathogens on healthcare resource utilization for pediatric inpatients. J Pediatr. 2016;173:196-201.

29. Rogers BB, Shankar P, Jerris RC, Kotzbauer D, Anderson EJ, Watson JR, et al. Impact of a rapid respiratory panel test on patient outcomes. Arch Pathol Lab Med. 2015;139(5):636-41.

30. The BioFire ${ }^{\circledR}$ FilmArray ${ }^{\circledR}$ Pneumonia Panel: Syndromic infectious disease testing for Pneumonia [Internet]. BIOFIRE A BIOMÈRIEUX COMPANY; c2019 [cited 2019 July 12]. Available from https://www.biofiredx.com/products/the-filmarray-panels/filmarray-pneumonia/ 31. Prospective Clinical Evaluation of the FilmArray Lower Respiratory Tract Infection (LRTI) Panel (LRTI) [Internet]. NIH U.S. National Library of Medicine; c2017 [cited 2019 July 12]. Available from https://clinicaltrials.gov/ct2/show/NCT03361670 
32. Pulido MR, Moreno-Martínez P, González-Galán V, Fernández Cuenca F, Pascual A, Garnacho-Montero J, et al. Application of Biofire FilmArray Blood Culture Identification panel for rapid identification of the causative agents of ventilator-associated pneumonia. Clin Microbiol Infect. 2018;24(11):e1-1213.e4.

33. Spina A, Kerr KG, Cormican M, Barbut F, Eigentler A, Zerva L, et al. Spectrum of enteropathogens detected by the FilmArray GI Panel in a multicenter study of communityacquired gastroenteritis. Clin Microbiol Infect. 2015;21:719-728

34. FDA 510(k) Substantial Equivalence Determination Decision Summary [Internet]. U.S. Food and Drug Administration, c2014 [cited 2019 July 12]. Available from https://www.accessdata.fda.gov/cdrh_docs/reviews/k140407.pdf

35. Buss SN, Leber A, Chapin K, Fey PD, Bankowski MJ, Jones MK, et al. Multicenter evaluation of the Biofire FilmArray gastrointestinal panel for etiologic diagnosis of infectious gastroenteritis. J Clin Microbiol. 2015;53(3):915-25.

36. Khare R, Espy MJ, Cebelinski E, Boxrud D, Sloan LM, Cunningham SA, et al. Comparative evaluation of two commercial multiplex panels for detection of gastrointestinal pathogens by use of clinical stool specimens. J Clin Microbiol. 2014;52(10):3667-73.

37. Gebrehiwot SA, Rucinski SL, Schwab JJ, Patel R, Snippes P. "Reflexive culture"-a strategy for laboratories adopting molecular testing for enteric pathogens. Poster 188. ASM Microbe 2016, Boston, MA

38. Shane AL, Mody RK, Crump JA, Tarr PI, Steiner TS, Kotloff K, et al. 2017 Infectious Diseases Society of America Clinical Practice Guidelines for the Diagnosis and Management of Infectious Diarrhea. Clin Infect Dis. 2017; 65(12):1963-1973. 
39. Giovane RA, Lavender PD. Central nervous system infections. Prim Care. 2018;45(3):505518.

40. Leber AL, Everhart K, Balada-Llasat JM, Cullison J, Daly J, Holt S, et al. Multicenter Evaluation of Biofire FilmArray Meningitis/Encephalitis Panel for Detection of Bacteria, Viruses, and Yeast in Cerebrospinal Fluid Specimens. J Clin Microbiol. 2016;54(9):2251-61.

41. Hanson KE, Slechta ES, Killpack JA, Heyrend C, Lunt T, Daly JA, et al. Preclinical assessment of a fully automated multiplex pcr panel for detection of central nervous system pathogens. J Clin Microbiol. 2016;54(3):785-787.

42. Launes C, Casas-Alba D, Fortuny C, Munoz-Almagro C, Valero-Rello A, Cabrerizo M. Utility of FilmArray Meningitis/Encephalitis Panel during Outbreak of Brainstem Encephalitis Caused by Enterovirus in Catalonia in 2016. J Clin Microbiol. 2017;55(1):336-338.

43. Messacar K, Breazeale G, Robinson CC, Dominguez SR. Potential clinical impact of the film array meningitis encephalitis panel in children with suspected central nervous system infections. Diagn Microbiol Infect Dis. 2016;86(1):118-20.

44. Graf EH, Farquharson MV, Cárdenas AM. Comparative evaluation of the FilmArray meningitis/encephalitis molecular panel in a pediatric population. Diagn Microbiol Infect Dis. 2017;87(1):92-94.

45. Schreckenberger PC, McAdam AJ. Point-counterpoint: Large Multiplex PCR Panels Should Be First-Line Tests for Detection of Respiratory and Intestinal Pathogens. J Clin Microbiol. 2015;53(10):3110-5.

46. Doern CD, Lacey D, Huang R, Haag C. Evaluation and implementation of FilmaArray version 1.7 for improved detection of adenovirus respiratory tract infection. J Clin Microbiol. 2013;51(12):4036-9. 
47. Midgley CM, Watson JT, Nix WA, Curns AT, Rogers SL, Brown BA, et al. Severe respiratory illness associated with a nationwide outbreak of enterovirus D68 in the USA (2014): A descriptive epidemiological investigation. Lancet Respir Med. 2015;3(11):879-87.

48. Michos A, Palili A, Koutouzis EI, Sandu A, Lykopoulou L, Syriopoulou VP. Detection of bacterial pathogens in synovial and pleural fluid with the FilmArray Blood Culture Identification System. IDCases. 2016;5:27-8.

49. Vasoo S, Cunningham SA, Greenwood-Quaintance KE, Mandrekar JN, Hanssen AD, Abdel MP, et al. Evaluation of the FilmArray Blood Culture ID Panel on Biofilms Dislodged from Explanted Arthroplasties for Prosthetic Joint Infection Diagnosis. J Clin Microbiol. 2015;53(8):2790-2792.

50. Fluit AC, Visser MR, Schmitz FJ. Molecular detection of antimicrobial resistance. Clin Microbiol Rev. 2001;14(4):836-71.

51. Milatovic D, Braveny I. Development of resistance during antibiotic therapy. Eur J Clin Microbiol. 1987;6(3):234-244

52. Deurenberg RH, Bathoorn E, Chlebowicz MA, Couto N, Ferdous M, Garcia-Cobos S, et al. Application of next generation sequencing in clinical microbiology and infection prevention. $\mathbf{J}$ Biotechnol. 2017;243:16-24

53. Ley RE, Ba $\square$ ckhed F, Turnbaugh P, Lozupone CA, Knight RD, Gordon JI. Obesity alters gut microbial ecology. Proc Natl Acad Sci U S A. 2005;102(31):11070-11075.

54. Qin J, Li R, Raes J, Arumugam M, Burgdorf KS, Manichanh C, et al. A human gut microbial gene catalogue established by metagenomic sequencing. Nature. 2010;464(7285):5965. 
55. Graessler J, Qin Y, Zhong H, Zhang J, Licinio J, Wong ML, et al. Metagenomic sequencing of the human gut microbiome before and after bariatric surgery in obese patients with type 2 diabetes: correlation with inflammatory and metabolic parameters. Pharmacogenomics J. 2013;13(6):514-522

56. Wang Z, Klipfell E, Bennett BJ, Koeth R, Levison BS, Dugar BS, et al. Gut flora metabolism of phosphatidylcholine promotes cardiovascular disease. Nature. 2011;472(7341):57-63.

57. Price KE, Hampton TH, Gifford AH, Dolben EL, Hogan DA, Morrison HG, et al. Unique microbial communities persist in individual cystic fibrosis patients throughout a clinical exacerbation. Microbiome. 2013;1(1):1-11.

58. Database for Reference Grade Microbial Sequences (FDA-ARGOS) [Internet]. U.S. Food and Drug Administration; c2018 [cited 2019 July 12]. Available from https://www.fda.gov/medical-devices/science-and-research-medical-devices/database-referencegrade-microbial-sequences-fda-argos

59. Rossen JWA, Friedrich AW, Moran-Gilad J, ESCMID Study Group for Genomic and Molecular Diagnostics (ESGMD). Practical issues in implementing whole-genome-sequencing in routine diagnostic microbiology. Clin Microbiol Infect. 2018;24(4):355-360.

60. Goldberg B, Sichtig H, Geyer C, Ledeboer N, Weinstock GM. Making the Leap from Research Laboratory to Clinic: Challenges and Opportunities for Next-Generation Sequencing in Infectious Disease Diagnostics. MBio. 2015;6(6):e01888-15.

61. Long SW, Williams D, Valson C, Cantu CC, Cernoch P, Musser JM, et al. A genomic day in the life of a clinical microbiology laboratory. J Clin Microbiol. 2013;51(4):1272-7. 
62. Fricke WF, Rasko DA. Bacterial genome sequencing in the clinic: Bioinformatic challenges and solutions. Nat Rev Genet. 2014;15(1):49-55.

63. Greninger AL. The challenge of diagnostic metagenomics. Expert Rev Mol Diagn. 2018;18(7):605-615

64. Novak SM, Marlowe EM. Automation in the clinical microbiology laboratory. Clin Lab Med. 2013;33(3):567-88.

65. Ledeboer NA, Dallas SD. The automated clinical microbiology laboratory: Fact or fantasy? J Clin Microbiol. 2014;52(9):3140-6.

66. Croxatto A, Prod'hom G, Faverjon F, Rochais Y, Greub G. Laboratory automation in clinical bacteriology: What system to choose? Clin Microbiol Infect. 2016;22(3):217-235.

67. Burckhardt I. Laboratory Automation in Clinical Microbiology. Bioengineering (Basel). 2018;5(4).pii:E102

68. Moreno-Camacho JL, Calva-Espinosa DY, Leal-Leyva YY, Elizalde-Olivas DC, CamposRomero A, Alcántar-Fernández J. Transformation from a conventional clinical microbiology laboratory to full automation. Lab Med. 2017;49(1):e1-e8.

69. Croxatto A, Dijkstra K, Prod'hom G, Greub G. Comparison of Inoculation with the InoqulA and WASP Automated Systems with Manual Inoculation. J Clin Microbiol. 2015;53(7):2298307.

70. Mischnik A, Trampe M, Zimmermann S. Evaluation of the impact of automated specimen inoculation, using Previ Isola, on the quality of and technical time for stool cultures. Ann Lab Med. 2015;35(1):82-88 
71. Froment P, Marchandin H, Vande Perre P, Lamy B. Automated versus manual sample inoculations in routine clinical microbiology: A performance evaluation of the fully automated InoqulA instrument. J Clin Microbiol. 2014;52(3):796-802.

72. Lainhart W, Burnham CA. Enhanced Recovery of Fastidious Organisms from Urine Culture in the Setting of Total Laboratory Automation. J Clin Microbiol. 2018;56(8).

Table 1: FDA-approved (non-panel) Molecular Tests per Organism/Organism Group

\begin{tabular}{|c|c|c|c|}
\hline Organism & $\begin{array}{c}\text { Number of } \\
\text { Tests }\end{array}$ & Organism & $\begin{array}{c}\text { Number of } \\
\text { Tests }\end{array}$ \\
\hline Adenovirus & 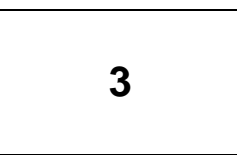 & $\begin{array}{l}\text { Influenza and } \\
\text { Respiratory } \\
\text { Viruses }\end{array}$ & 50 \\
\hline Bacillus anthracis & 1 & Leishmania spp. & 1 \\
\hline Bordetella spp. & 5 & $\begin{array}{l}\text { Mycobacterium } \\
\text { tuberculosis }\end{array}$ & 8 \\
\hline Candida spp. & 6 & $\begin{array}{l}\text { Mycobacterium } \\
\text { spp. }\end{array}$ & 9 \\
\hline Clostridium difficile & 17 & $\begin{array}{l}\text { Mycoplasma } \\
\text { genitalium }\end{array}$ & 1 \\
\hline Coxiella burnetii & 1 & $\begin{array}{l}\text { Mycoplasma } \\
\text { pneumoniae }\end{array}$ & 2 \\
\hline $\begin{array}{l}\text { Chlamydia } \\
\text { trachomatis/Neisseria } \\
\text { gonorrhoeae }\end{array}$ & 47 & $\begin{array}{l}\text { Non-Variola } \\
\text { Orthopoxvirus }\end{array}$ & 1 \\
\hline Cytomegalovirus & 5 & Norovirus & 1 \\
\hline Dengue virus & 1 & Plasmodium spp. & 1 \\
\hline Enterococcus spp. & 6 & Rickettsia spp. & 1 \\
\hline Enterovirus & 1 & Staphylococci & 19 \\
\hline $\begin{array}{l}\text { Escherichia coli/Klebsiella } \\
\text { pneumoniae/Pseudomonas } \\
\text { aeruginosa }\end{array}$ & 1 & Streptococci & 21 \\
\hline Francisella tularensis & 1 & Shiga toxin & 1 \\
\hline Herpes Simplex Virus & 18 & $\begin{array}{l}\text { Trichomonas } \\
\text { vaginalis }\end{array}$ & 7 \\
\hline Hepatitis Virus & 14 & Variola & 1 \\
\hline Human Metapneumovirus & 3 & Yersinia pestis & 1 \\
\hline Human papillomavirus & 7 & & \\
\hline
\end{tabular}

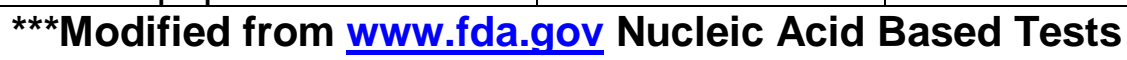




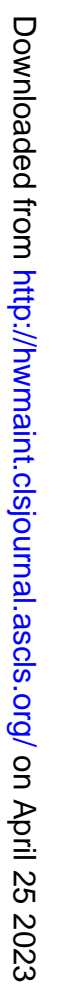

